\title{
Coronavirus Disease 2019 (COVID-19) in Children - What We Know So Far and What We Do Not
}

\author{
S Balasubramanian ${ }^{1}$, Neha Mohan RaO ${ }^{2}$, AnU Goenka ${ }^{3}$, Marion Roderick ${ }^{4}$ and Athimalaipet V Ramanan ${ }^{5}$ \\ ${ }^{1}$ Department of Pediatrics, Kanchi Kamakoti Childs Trust Hospital, Chennai, India; ${ }^{2}$ Consultant, Bangalore, India; ${ }^{3}$ Paediatric \\ Immunology and Infectious Diseases Service, Bristol Royal Hospital for Children, and Bristol Children's Vaccine Centre, Schools of \\ Cellular and Molecular Medicine and of Population Health Sciences, University of Bristol, UK; ${ }^{4}$ Pediatric Immunology and \\ Infectious Diseases Service, Bristol Royal Hospital for Children, UK; and ${ }^{5}$ Department of Pediatric Rheumatology, Bristol Royal \\ Hospital for Children, UK and Translational Health Sciences, University of Bristol, UK. \\ Correspondence to:Dr. Neha Mohan Rao, 588, 7th Main, 17th Cross, Indiranagar, Second stage, Bangalore 560038, Karnataka, \\ India.neharao89@gmail.com
}

Pediatric coronavirus disease-19 (COVID-19) infection is relatively mild when compared to adults, and children are reported to have a better prognosis. Mortality in children appears rare. Clinical features of COVID-19 in children include fever and cough, but a large proportion of infected children appears to be asymptomatic and may contribute to transmission. It remains unclear why children and young adults are less severely affected than older individuals, but this might involve differences in immune system function in the elderly and/or differences in the expression/function of the cellular receptor for Severe Acute Respiratory Syndrome Coronavirus 2 (SARS-CoV2)- Angiotensin converting enzyme 2 (ACE2). Laboratory findings and chest imaging may not be specific in children with COVID-19. Diagnosis is by Reverse transcriptase-Polymerase chain reaction (RT-PCR) testing of upper or lower respiratory tract secretions. This review additionally considers COVID-19 in immunosuppressed children, and also suggests a management algorithm for the few children who appear to present with life threatening infection, including the potential use of antiviral and immunomodulatory treatment. The most significant threat to global child health from SARS-CoV-2 is unlikely to be related to COVID 19 in children, but rather the socio-economic consequences of a prolonged pandemic.

Keywords: SARS-CoV-2, Pandemic, Management, Immunosuppressed.

Published online: April 09, 2020; PII: S0997475591600154

$\mathrm{C}$ oronavirus disease-2019 (COVID-19) is a global health crisis. The clinical characteristics, disease progression and outcome in children and young adults appear significantly milder compared to older individuals. Since first being reported in Wuhan, China in December 2019, COVID-19 has rapidly spread to affecting over 200 countries worldwide. Children account for $1-5 \%$ of diagnosed COVID-19 cases [1]; although, many infected children may be asymptomatic and therefore not diagnosed without population screening. At the time of writing, in India, the number of virologically confirmed COVID-19 positive cases is 5273 (149 deaths) as per the Ministry of Health and Family Welfare (MOHFW) [2].

\section{VIROLOGY AND EPIDEMIOLOGY}

Coronaviruses are a family of enveloped, single stranded, zoonotic RNA viruses which can rapidly mutate and recombine, leading to novel viruses that can spread from animals to humans [3]. The precise events that led to the emergence of Severe Acute Respiratory Syndrome Coronavirus-2 (SARS-CoV-2) causing COVID-19 remain unknown. SARS-CoV-2 is transmitted through inhalation of respiratory droplets of an infected person and touching surfaces contaminated with the virus. In previous coronavirus epidemics, children globally accounted for $6.9 \%$ of SARS 2002-3 infections and 2\% of Middle East Respiratory Syndrome (MERS) infections. It appears that SARS-CoV-2 has a higher transmission capability compared with the closely related viruses causing SARS 2002-3 and MERS [4]. The true case fatality rate (CFR) of COVID-19 infection is currently unknown due to lack of population-scale longitudinal data. Thus, estimates of CFR currently vary between $0.5-5 \%[1,5]$.

\section{PATHOGENESIS}

The SARS-CoV-2 virus utilizes Angiotensin Converting Enzyme 2 (ACE2) receptors as its cell surface receptor, similar to the SARS 2002-3 virus. ACE2 is expressed in highly byciliated epithelial cells in the human lungs and this receptor allows the virus to attach to the cell [6]. The ACE2 receptor is also expressed in the intestines, potentially accounting for the gastrointestinal symptoms that commonly occur in the early stage of the illness. 
Severe COVID-19 disease is characterized by three phases: the first being the viral phase; the second being the cytokine storm; and the third encompassing acute respiratory distress syndrome (ARDS), impaired cardiac function and death [7]. The cytokine storm appears to be driven by a dysregulated host immune response [8] and might contribute to mortality [9]. The profile of the cytokine storm associated with severe COVID-19 disease is similar to that of secondary hemophagocytic lymphohistiocytosis (HLH), which is a rare complication of other viral infections (3.7-4.3\%) [8]. Secondary HLH is characterized by fulminant and fatal hypercytokinemia with multiorgan failure. In severe infection, lower peripheral lymphocyte counts (CD4 and CD8 T cells), higher interleukin (IL) levels (IL-6 and IL-10), decreased interferon-gamma expression in $\mathrm{CD} 4+\mathrm{T}$ cells and higher D-dimer and fibrin degradation products (FDP) levels, leading to increased thrombosis and multiorgan injury has been described. Moreover, patients with severe infection may also have abnormal coagulation parameters, perhaps related to high expression of ACE2 receptors in vascular endothelial cells.

\section{TRANSMISSION}

Most infected children are likely to be secondary cases and acquire the infection after exposure to a COVID-19 positive adult, although there are no longitudinal data to confirm this yet. Intra-family transmission may be important [10]. An as yet unquantified proportion of children with COVID-19 is asymptomatic and may contribute to transmission. It is unknown whether COVID-19 is acquired by contact with infected feces $[10,11]$. In a report of 10 children admitted for COVID-19 with positive nasopharyngeal swabs, 8 of 10 children demonstrated persistently positive real time reverse transcriptase-polymerase chain reaction (RT-PCR) of rectal swabs after their nasopharyngeal testing had become negative [12]. It remains unclear whether the detection of virus by RT-PCR in fecal matter represents active viral replication or residual viral genomic material; however, it appears that viral shedding from the digestive tract might be greater and last longer than that from the respiratory tract [12].

\section{Why is Covid-19 Milder in Children?}

Multiple reports have demonstrated that children and young adults have a milder form of the disease compared to adults [13]. Asymptomatic, mild and moderate infections comprise over $90 \%$ of all children who have tested positive for COVID-19 with fewer severe and critical cases (5.9\%) compared to adults (18.5\%) [13]. The possible reasons for lower number and milder infections in children and young adults include lower exposure to virions, being isolated at home and minimal exposure to pollution and cigarette smoke contributing to healthier respiratory tracts. The elderly may be susceptible to severe COVID-19 disease by their qualitatively different immune response, encompassed by the terms 'immunosenescence' and 'inflammaging' [14]. Viral co-infection may be important in potentially leading to limited replication of the SARS-CoV-2 by direct virus-to-virus interaction and competition [15]. Additionally, the distribution, maturation and functioning of viral receptors such as ACE2 may be important in age-dependent susceptibility to severe COVID-19 [13,16].

Due to smaller number of reported cases in children, it is at present challenging to delineate the clinical characteristics of children with severe COVID-19 infection, combined with the lack of a clear biomarker to indicate severity of infection [17]. Dong, et al. [13], in the largest pediatric review of 2143 children, described that $13 \%$ of virologically confirmed children were asymptomatic. This makes epidemiological inference problematic since asymptomatic children are less likely to be tested and may still contribute to transmission. In addition, a significant proportion of children can also have coinfections with other viruses, and the detection of SARSCoV-2 may therefore be clinically insignificant [11]. It has been proposed that the outcome for some children may be worse due to exposure to antenatal smoking and obesity [17].

Another theory that has been postulated is the protective role of Bacillus Calmette-Guérin (BCG) vaccine in COVID-19. BCG vaccination has been associated with heterologous immunity to other pathogens, potentially by a phenomenon called 'trained immunity' involving innate cells such as macrophages, monocytes and epithelia [18]. Trials are underway to understand if BCG vaccination may offer protection against COVID-19.

\section{CLINICAL FEATURES}

Children of all ages can be infected with COVID-19, with more cases reported in younger children and infants [13]. Acknowledging the possible reporting biases discussed above, there is no age or sex preponderance [13] and the median age of infection is 6.7 years (rangenewborn to 15 years) [19]. The incubation period of COVID-19 in children has been reported as 2 days (range-2 to 10 days) [1]. At the time of diagnosis, 13$15 \%$ of virologically positive children may be asymptomatic $[13,19]$. The most common symptoms described at onset in children are fever $(50 \%)$ and mild cough (38\%) [10]. Fever is present in about $40 \%$ of 
children [19]. Other clinical features include sore throat, rhinorrhea, sneezing, myalgia, fatigue, diarrhea and vomiting. Children may have more upper respiratory symptoms than lower respiratory symptoms [13], and appear to recover in 1-2 weeks [20].

\section{Risk Stratification and Severity Classification}

In the largest pediatric cohort to date, Dong, et al. [13] describe suspected and confirmed cases based on symptoms, laboratory abnormalities, chest imaging, and RT-PCR/genomic analysis. The severity of COVID-19 was divided into asymptomatic, mild, moderate, severe and critical. Severe COVID-19 accounted for 18 (2.5\%) of virologically confirmed cases, and furthermore the definition of severe included children with only mild hypoxia. Critical COVID-19 was observed in $3(0.4 \%)$ of virologically confirmed cases, defined by the presence of ARDS or organ failure. Though data on chronology of complications and predictors of mortality is available in adults, there is insufficient data on predictors of mortality in children.

\section{DIAGNOSTIC TECHNIQUES}

The Ministry of Health and Family Welfare (MOHFW) [2] in their updated guidelines (as of 7 April, 2020) has categorized patients into three groups - those with mild, moderate and severe illness, and have designated COVID dedicated facilities for their treatment.

RT-PCR testing of nose and throat swab for detection of SARS-CoV-2 nucleic acid has been recommended as the confirmatory test for COVID-19 [21]. Other alternative samples for RT-PCR include bronchoalveolar lavage or endotracheal aspirate. The Government of India has now advised the use of antibody tests in patients with symptomatic influenza-like illness (ILI) in 25 districts across the country, or 'COVID hotspots' [22]. Based on the results of the antibody test, confirmatory RT-PCR and clinical assessment, hospital treatment or home isolation measures are instituted, with contact tracing measures as per protocol.

The limited data in children describes relatively lower rates of lymphopenia and elevated inflammatory markers compared to adults [1]. Henry, et al. [23] summarized the findings from 12 studies on 66 children and reported normal leucocyte counts (69.2\%), neutropenia (6.0\%), neutrophilia $(4.6 \%)$ and lymphopenia (3.0\%). C-reactive protein (CRP) and procalcitonin were high only in $13.6 \%$ and $10.6 \%$ of cases, respectively. Slight elevation of liver transaminases is common [23]. It is recommended to monitor the lymphocyte count and CRP as signs for severe infection, while using procalcitonin levels to detect potential bacterial co-infection [23].

Chest $X$-ray findings in children appear to be nonspecific. Children with mild disease should not routinely need computed tomography (CT) chest imaging in view of the high radiation exposure [24]. When CT is performed, ground glass opacities is seen in one third of patients [19]. Peripheral distribution of lung lesions has been noted, with multilobar involvement [25]. Consolidation with surrounding halo sign is considered typical of pediatric patients [26]. However, chest CT alone cannot accurately diagnose COVID-19 due to similar radiological presentations with other infections.

Patients admitted with severe infection are known to have elevated plasma levels of IL-2, IL-7, IL-10, granulocyte colony stimulating factor (GCSF), interferon-gamma-inducible protein 10 (IP10), monocyte chemoattractant protein 1(MCP1), macrophage inflam-matory protein 1-alpha (MIP1A) and tumor necrosis factor (TNF) alpha [9]. In a study comprising of 150 confirmed COVID-19 cases in Wuhan, China, elevated ferritin (mean $1298 \mathrm{ng} / \mathrm{mL}$ vs $614 \mathrm{ng} / \mathrm{mL} ; P<0.001)$ and IL-6 levels $(P<0.0001)$ were found in survivors compared to non-survivors [7]. These cytokines are produced by inflammatory macrophages which have been implicated in the cytokine storm. This is similar to previous outbreaks of MERS and SARS 2002-3 in terms of having high proinflammatory cytokines in patients with severe disease [27].

\section{MANAGEMENT OF PEDIATRIC COVID-19}

Upon suspicion of COVID-19 infection, immediate Infection prevention control (IPC) measures must be instituted. Standard precautions such as hand hygiene, use of personal protective equipment (PPE), safe waste management and cleaning and disinfection of equipment must be followed as per the guidelines issued by the MOHFW [2].

For the few children who will require admission to a healthcare facility, the cornerstone of management is supportive therapy including adequate nutrition and calorie intake, fluid and electrolyte management and oxygen supplementation. Communication with parents and alleviating anxiety is an important part of management. In adults with severe COVID-19, early intubation and mechanical ventilation with lung protective strategies and prone positioning has been recommended [20]. Antibiotics may be indicated if bacterial super-infection is suspected.

There are no randomized clinical trial data to guide treatment of the very few children that present with lifethreatening COVID-19 including severe pneumonia, 
ARDS, sepsis and septic shock. Hence, the World Health Organization has not recommended any specific treatment for children until the results of ongoing clinical trials are available. We strongly believe that clinical trials of all therapeutic agents for COVID-19 are needed in children as well. It is important that when such clinical trials are open, children are treated only in the context of clinical trials and not outside these. In the absence of data from these trials, clinicians may be left in the difficult scenario of deciding whether to pursue treatment with antiviral drugs and immunomodulatory therapies for children with severe COVID-19. A relatively new antiviral drug being tested in adults with COVID-19 is remdesivir, which in combination with chloroquine has been found to inhibit SARS-CoV-2 growth in vitro [28]. Interferon alpha- $2 \mathrm{~b}$ and oral lopinavir/ritonavir together with corticosteroids for complications and intravenous immunoglobulin for severe cases has been recommended in one report in China [29]. A HIV test should be performed before commencing antiviral treatment, in particular lopinavir/ritonavir.

The MOHFW has allowed off label use of hydoxychloroquine in combination with azithromycin in adults with severe disease and requiring intensive care [2].
However, these treatments are not currently recommended in children below the age of 12 years. Corticosteroids are not routinely recommended and might exacerbate COVID-19 associated lung injury [30]. Ivermectin, the broad spectrum anti-parasitic agent, has in vitro antiviral action against SARS-CoV-2 [31].

Owing to the cytokine storm syndrome in COVID-19, there may potentially be a role of immunomodulators in treating patients with severe infections to ameliorate pulmonary inflammation and hopefully improve mortality. There is an established role of anakinra (IL-1 blockade) in survival benefit of patients with hyperinflammation, without increased adverse events [8]. A multicenter randomized control trial (RCT) of the IL-6 receptor blocker, tocilizumab is in progress in China for adults with COVID-19 pneumonia and raised IL-6 levels (ChiCTR2000029765) [32]. There may also potentially be a role of janus kinase inhibitors (JKI), since these drugs block downstream inflammatory pathways and may alter cellular viral entry [33].

A suggested management algorithm based on the limited observational data from adults is depicted in Figs. 1 and 2. The common drugs used in COVID-19 are detailed in Table I. It is important to note that very few

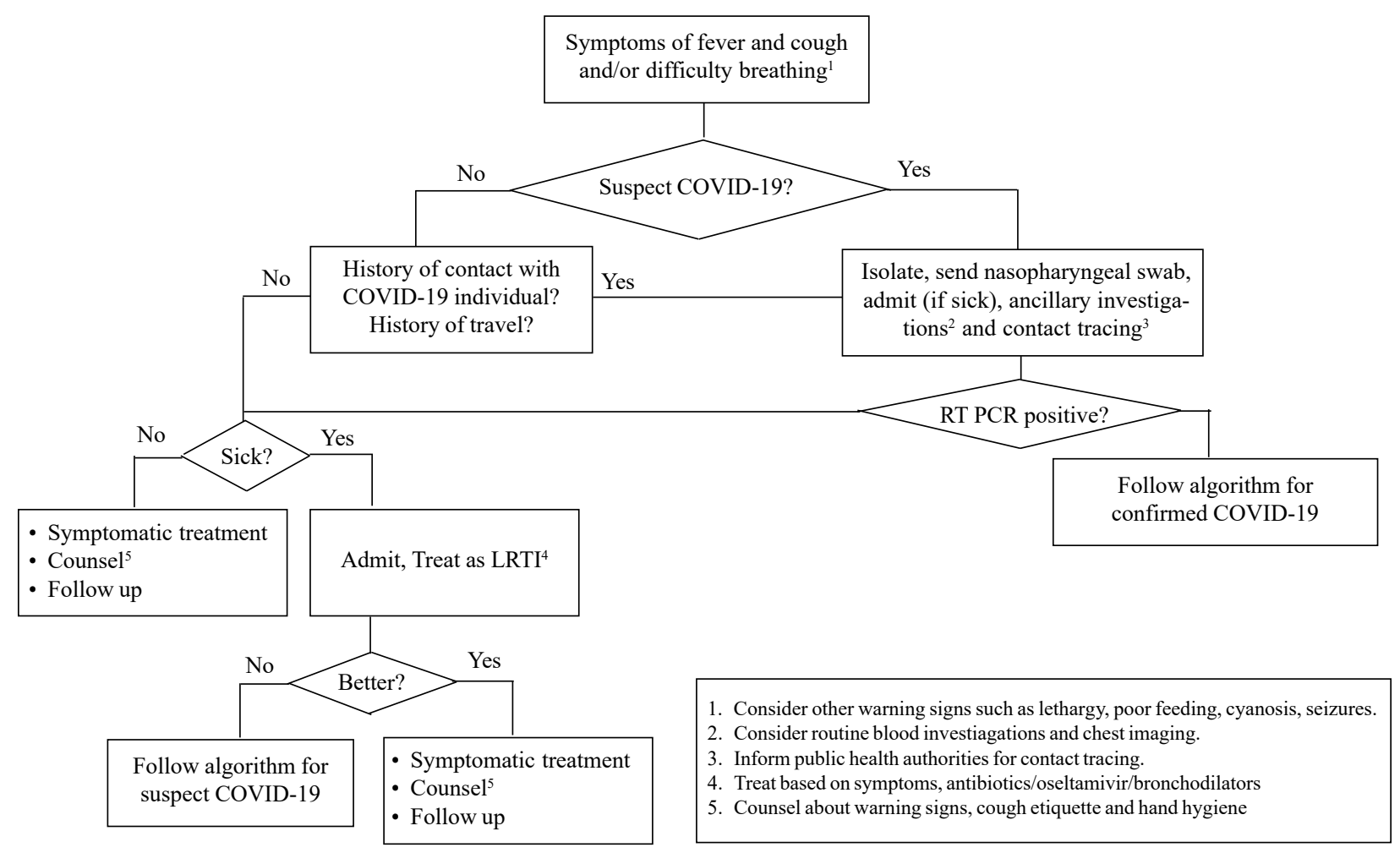

Fig.1 Suggested algorithm for case management of children with COVID-19 symptoms. 


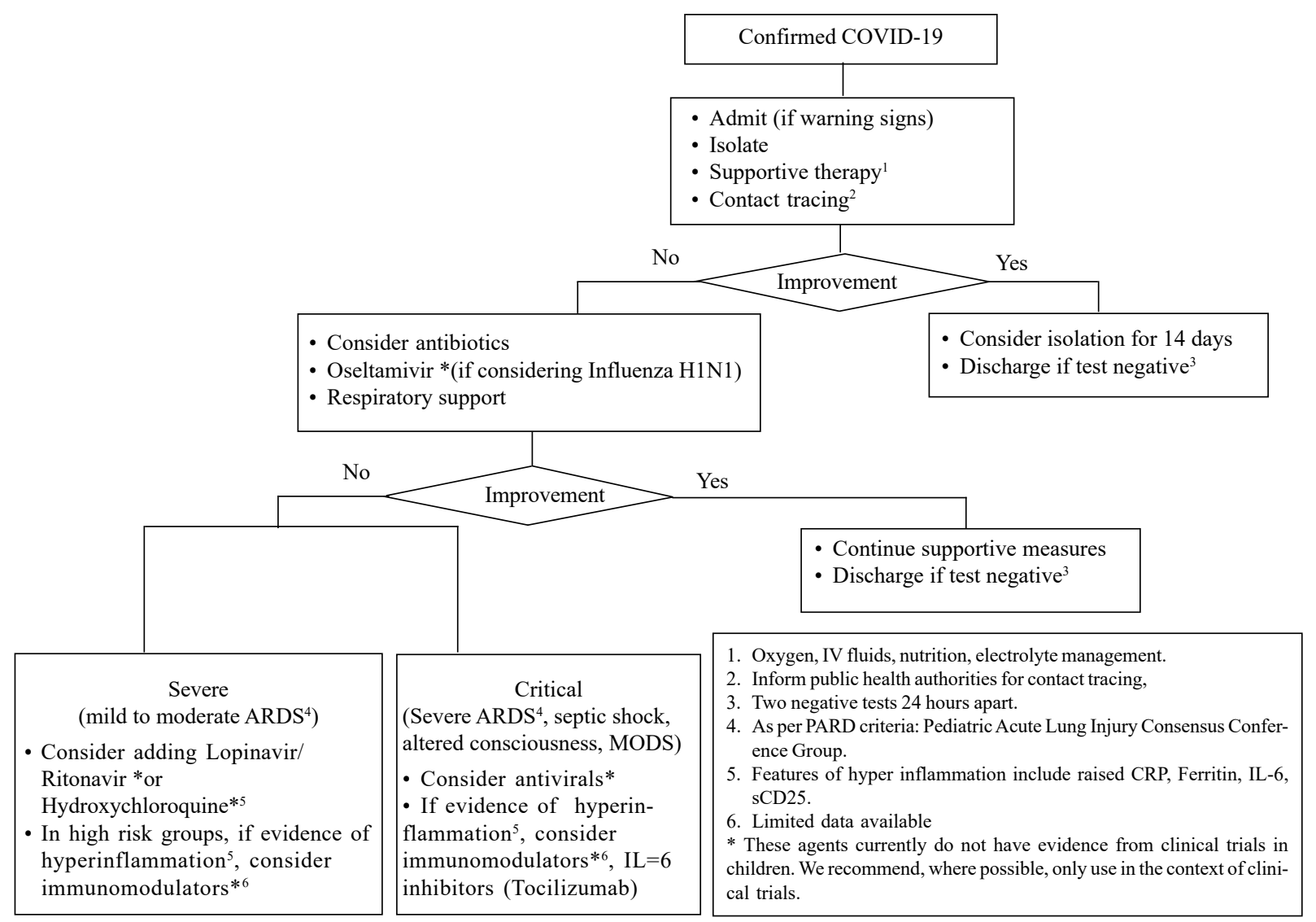

Fig. 2 Suggested algorithm for case management of confirmed COVID-19 (Adapted from the BPAIIG Position Statement: SARS CoV2 Treatment Guidance version 1.2) [37].

Table I Medications for Coronavirus Disease 2019 (Covid-19)

\begin{tabular}{ll}
\hline Drug & Indication/Limitation \\
\hline Paracetamol & - Recommended antipyretic (Avoid ibuprofen) \\
Oseltamivir & - To be considered in influenza $\mathrm{H}_{1} \mathrm{~N}_{1}$ is differential \\
Lopinavir/Ritonavir & - Can be considered if no improvement/severe infection \\
& - Do not co-administer with hydroxychloriquine \\
Remdesivir & - Limited data available on benefit in children \\
Hydroxychloroquine & - Can be used if no improvment/severe infection in children $>12$ years \\
& - Do not co-administer with azithromycin \\
Azithromycin & - Limited data available on benefit in children \\
Ivermectin & Can be considered if no improvment/severe infection \\
Immunomodulators & Limited data available on benefit in children \\
- $1 \mathrm{~L} 1$ inhibitor (Anakinra) & Has shown in vitro anti-viral activity \\
\hline
\end{tabular}


children with COVID-19 are likely to need any specific therapy other than supportive treatment, and the decision to start antiviral or immunomodulatory treatment should therefore be made carefully in consultation with experts in pediatric infectious disease and immunology. Given that severe COVID-19 appears very rare in children, an important part of this assessment is ascertaining whether a positive RT-PCR for SARS-CoV-2 is a clinically important factor in explaining the child's condition, or whether more occult pathology may be responsible.

For neonatal management of COVID-19 infected mothers, it is recommended to have a separate room adjacent to the delivery room for neonatal resuscitation or for resuscitation staff to maintain atleast a 2 meter gap between the infected mother and newborn [34]. Only essential personnel should attend the delivery with full PPE, with the mother following meticulous hand hygiene and wearing a mask. Standard neonatal resuscitation measures are to be followed and positive pressure ventilation if needed should be provided by a selfinflating bag and mask rather than a T-piece resuscitator. If the baby requires intensive care, a single patient room is ideal preferably with negative pressure. The baby should be tested at 24 hours of life and repeat testing should be performed at 48 hours. Antivirals/hydroxychloroquine/steroids or intravenous immunoglobulin (IVIG)should not be administered to the newborn. The baby should then be tested every 48-72 hours until two consecutive negative tests. It is critical that breastfeeding shouldbe encouraged with the mother wearing a mask. The baby should be vaccinated prior to discharge from the hospital.

\section{IMPACT ON IMMUNOSUPPRESSED CHILDREN}

Data on children with immunocompromised conditions and COVID-19 are scarce, but severe disease may be more common in adults with cancer [35]. Despite concerns that immunocompromised children may have severe infection analogous to infection with adenovirus, rhinovirus, influenza, respiratory syncytial virus, and experience from previous pandemics (such as influenza H1N1), Antiga, et al. [36] described that children who were immunocompromised were not at greater risk of severe COVID-19, probably owing to the fact that a functional host innate immune response is the main driver for lung damage. In Bergamo, among 200 transplant recipients including 10 inpatients, 100 with autoimmune liver disease and three undergoing chemotherapy for hepatoblastoma (inpatients), none had clinical pulmonary disease, despite the fact that 3 patients tested positive for SARS-CoV-2, suggesting that the immunocompromised may be protected by their weaker immune response. No data is available on severity of COVID-19 infection in children with malnutrition, rheumatic heart disease or Human Immunodeficiency Virus (HIV) positive children.

\section{THE FUTURE}

Several vaccines against SARS-CoV-2 are in development; however, it remains unclear when a successful vaccine might be rolled out. Studies on factors responsible for immune dysregulation may provide insights into developing vaccines capable of inducing durable protective immunity and avoiding vaccinerelated adverse events.

This unprecedented pandemic should prompt improved global surveillance of infectious diseases, as well as cooperation and communication so that the global society remains interconnected and limits the spread of this outbreak.

Lastly, we fear the greatest impact on children from COVID-19 is likely to be delayed presentation of other childhood illnesses due to fear and ignorance amongst parents/families. This coupled with the impact of economic uncertainty on those in the low socioeconomic strata, is likely to have a greater adverse impact on child health in India in these uncertain times.

Contributors: SB, AVR- Initiated the preparation of the manuscript;NMR: Substantial contribution to the conception and design of the work, and prepared and finalized the draft; SB, AVR, AG, MR-Substantial contributions to the acquisition, analysis, and interpretation of data for the work, SB, AVR, AG, MR-Revising it critically for important intellectual content;SB, NMR, AG, MR, AVR: Final approval of the version to be published, and agreement to be accountable for all aspects of the work in ensuring that questions related to the accuracy or integrity of any part of the work are appropriately investigated and resolved.

Funding: None; Competing interests: None stated.

\section{REFERENCES}

1. Ludvigsson JF. Systematic review of COVID-19 in children show milder cases and a better prognosis than adults. Acta Paediatr. 2020 Mar 23. [Epub ahead of print]https://onlinelibrary.wiley.com/doi/epdf/10.1111/ apa.15270. Accessed on April 08, 2020.

2. Ministry of Health and Family Welfare. Available from: https://www.mohfw.gov.in. Accessed on April 08,2020.

3. Zimmermann P, Curtis N. Coronavirus Infections in Children Including COVID-19. PIDJ. 2020 Mar 12. [Epub ahead of print]Available from: https://journals.lww.com/ pidj/Abstract/onlinefirst/Coronavirus_Infections_in Children_Including.96251.aspx.Accessed on April 06, 2020.

4. Pedersen SF, Ho Y. SARS-CoV-2/ : A Storm is Raging. JCI. 2020 Mar 27. [Epub ahead of print]. Available from: 
https://www.jci.org/articles/view/137647/pdf. Accessed on April 05, 2020.

5. Spychalski P, Blazynska-Spychalska A, Kobiela J. Estimating case fatality rates of COVID-19.Lancet Infect Dis. 2020 Mar 31. [Epub ahead of print]Available from: https://www.thelancet.com/pdfs/journals/laninf/PIIS14733099(20)30246-2.pdf. Accessed on April 04, 2020.

6. Weston S, Frieman MB. COVID-19: Knowns, Unknowns, and Questions. mSphere. 2020 Mar 18. [Epub ahead of print]. Available from: https://msphere.asm.org/content/ msph/5/2/e00203-20.full.pdf. Accessed on April 07, 2020.

7. Brodin P. Why is COVID-19 so mild in children? Acta Paediatr. 2020 Mar 25. [Epub ahead of print]. Available from: https://onlinelibrary.wiley.com/doi/epdf/10.1111/ apa.15271. Accessed on April 06, 2020.

8. Mehta P, Mcauley DF, Brown M, Sanchez E, Tattersall RS, Manson JJ, et al. COVID-19: consider cytokine storm syndromes and immunosuppression. Lancet. 2020; 395: 1033-4.

9. Huang C, Wang Y, Li X, Ren L, Zhao J, Hu Y, et al. Clinical features of patients infected with 2019 novel coronavirus in Wuhan, China. Lancet. 2020; 395:497506.

10. Jiehao C, Jing X, Daojiong L, Lei X, Zhenghai Q, Yuehua $\mathrm{Z}$, et al. A case series of children with 2019 novel coronavirus infection: clinical and epidemiological features. CID. 2020 Feb 28. [Epub ahead of print]. Available from: https://academic.oup.com/cid/advancearticle-pdf/doi/10.1093/cid/ciaa198/32709823/ ciaa198.pdf. Accessed on April 06, 2020.

11. Cruz A, Zeichner S. COVID-19 in Children: Initial characterization of the pediatric disease. Pediatrics. 2020 Mar. [Epub ahead of print]. Available from: https:// pediatrics.aappublications.org/content/pediatrics/early/ 2020/03/16/peds.2020-0834.1.full-text.pdf. Accessed on April 06, 2020.

12. Xu Y, Li X, Zhu B, Liang H, Fang C, Gong Y, et al. Characteristics of pediatric SARS-CoV-2 infection and potential evidence for persistent fecal viral shedding. Nat Med. 2020 Mar 13. Available from: https:// www.nature.com/articles/s41591-020-0817-4.pdf. Accessed on April 05, 2020. [Epub ahead of print]

13. Dong Y, Mo X, Hu Y, Qi X, Jiang F, Jiang Z, et al. Epidemiological characteristics of 2143 pediatric patients with 2019 coronavirus disease in China. Pediatrics. 2020 Mar. Available from: https://pediatrics.aappublications. org/content/pediatrics/early/2020/03/16/peds.20200702.1.full-text.pdf. Accessed on April 04, 2020. [Epub ahead of print]

14. Murray MA, Chotirmall SH. The impact of immunosenescence on pulmonary disease. Hindawi [Internet]. 2015 Jun 24. Available from: http:// downloads.hindawi.com/journals/mi/2015/692546.pdf. Accessed on April 06, 2020.

15. Nickbakhsh S, Mair C, Matthews L, Reeve R, Johnson PCD, Thorburn F, et al. Virus-virus interactions impact the population dynamics of influenza and the common cold. PNAS [Internet]. 2019 Dec 26;116:27142-50. Available from:https:/www.pnas.org/content/pnas/116/52/27142. full.pdf. Accessed on April 04, 2020.

16. Lee PI, Hu YL, Chen PY, Huang YC, Hsueh PR. Are children less susceptible to COVID-19? JMII [Internet]. 2020 Feb 25;S1684-1182(20)30039-6. Available from: https://www.ncbi.nlm.nih.gov/pmc/articles/PMC7102573/ pdf/main.pdf. Accessed on April 04, 2020.

17. Sinha IP, Harwood R, Semple MG, Hawcutt DB, Thursfield R, Narayan O, et al. COVID-19 infection in children. Lancet Respir. 2020 Mar 27. Available from: https://www.thelancet.com/action/showPdf?pii=S22132600\%2820\%2930152-1. Accessed on April 05, 2020. [Epub ahead of print]

18. Covián C, Fernández-Fierro A, Retamal-Díaz A, Díaz FE, Vasquez AE, Lay MK, et al. BCG-induced cross-protection and development of trained immunity: Implication for vaccine design.Front Immunol [Internet]. 2019 Nov 29;10:2806. Available from: https://www.ncbi.nlm. nih.gov/pmc/articles/PMC6896902/pdf/fimmu-1002806.pdf. Accessed on April 04, 2020.

19. Lu X, Zhang L, Du H, Zhang J, Li YY, Qu J, et al. SARSCoV-2 Infection in children. NEJM. 2020 Mar 18. Available from: https://www.nejm.org/doi/pdf/10.1056/ NEJMc2005073? articleTools=true. Accessed on April 05, 2020. [Epub ahead of print]

20. Cao Q, Chen Y, Chen C, Chiu C. SARS-CoV-2 Infection in children: Transmission dynamics and clinical charateristics. J Formos Med Assoc. 2020;119:670-673.

21. Revised Guidelines on Clinical Management of COVID 19 [Internet]. Ministry of Health and Family Welfare. Available from: https://www.mohfw.gov.in/pdf/ RevisedNationalClinicalManagementGuidelinefor COVID1931032020.pdf. Accessed April 01, 2020.

22. Advisory to start rapid antibody based blood test for COVID-19 [Internet]. Indian Council of Medical Research. Available from: https://www.mohfw.gov.in/pdf/ Advisory\&StrategyforUseofRapidAntibodyBasedBloodTest. pdf. Accessed April 05, 2020.

23. Henry BM, Lippi G, Plebani M. Laboratory abnormalities in children with novel coronavirus disease 2019. CCLM. 2020 Mar 16. Available from: https://www.degruyter.com/ downloadpdf/journals/cclm/ahead-of-print/article10.1515-cclm-2020-0272/article-10.1515-cclm-20200272.xml. Accessed on April 05, 2020. [Epub ahead of print]

24. Kelvin AA, Halperin S. COVID-19 in children: the link in the transmission chain. Lancet Infect Dis. 2020 Mar 25. Available from: https://www.thelancet.com/action/ showPdf?pii=S1473-3099\%2820\%2930236-X. Accessed on April 06, 2020. [Epub ahead of print]

25. Li B, Shen J, Li L, Yu C. Radiographic and Clinical Features of Children with 2019 Novel Coronavirus (COVID-19) Pneumonia. Indian Pediatr. 2020 Apr 07. Available from: https://www.indianpediatrics.net/ CONVID29.03.2020/RP-00156.pdf Accessed on April 08, 2020. [Epub ahead of print]

26. Xia W, Shao J, Guo Y, Peng X, Li Z, Hu D. Clinical and CT features in pediatric patients with COVID-19 infection: Different points from adults. Pediatric Pulmonology. 2020 Mar 5. Available from: https://onlinelibrary.wiley.com/ 
doi/epdf/10.1002/ppul.24718. Accessed on April 05, 2020. [Epub ahead of print]

27. Mahallawi W, Khabour O, Zhang Q, Makhdoum H, Suliman B. MERS-CoV infection in humans is associated with a pro-inflammatory Th1 and Th17 cytokine profile. Cytokine. 2018;104:8-13.

28. Wang M, Cao R, Zhang L, Yang X, Liu J, Xu M, et al. Remdesivir and chloroquine effectively inhibit the recently emerged novel coronavirus (2019-nCoV) in vitro. Cell Res. 2020;30:269-71.

29. Qiu H, Wu J, Hong L, Luo Y, Song Q, Chen D. Clinical and epidemiological features of 36 children with coronavirus disease 2019 (COVID-19) in Zhejiang, China: an observational cohort study. Lancet Infect Dis. 2020 Mar 25. Available from: https://www.thelancet.com/action/ showPdf?pii=S1473-3099\%2820\%2930198-5. Accessed on April 05, 2020. [Epub ahead of print]

30. Russel CD, Millar JE, Baillie JK. Clinical evidence does not support corticosteroid treatment for 2019-nCoV lung injury. Lancet. 2020;395:473-5.

31. Caly L, Druce JD, Catton MG, Jans DA, Wagstaff KM. The FDA-approved Drug Ivermectin inhibits the replication of SARS-CoV-2 invitro. Antiviral Res. 2020 Apr 03. Available from: https://www.sciencedirect.com/science/article/pii/ S0166354220302011/pdfft? md5=bd2a8d1cfbe3680f $2 d 40$ 5b4a62642a15\&pid=1-s2.0-S0166354220302011-main. $p d f$. Accessed on April 05, 2020. [Epub ahead of print]

32. Chinese Clinical Trial Registry. A multicenter, randomized control trial for the efficacy and safety of tocilizumab in the treatment of new coronavirus pneumonia (COVID-19).
2020 Feb 13. Available from: http://www.chictr.org.cn/ showprojen.aspx?proj=49409. Accessed April 05, 2020.

33. Richardson P, Griffin I, Tucker C, Smith D, Oechsle O, Phelan A, et al. Baricitinib as potential treatment for 2019nCoV acute respiratory disease. Lancet. 2020;395:E30E31. Available from: https://www.thelancet.com/action/ showPdf?pii=S0140-6736\%2820\%2930304-4. Accessed on April 05, 2020.

34. Chawla D, Chirla D, Dalwai S, Deorari AK, Ganatra A, Gandhi A, et al. Perinatal-Neonatal Management of COVID-19 Infection - Guidelines of the Federation of Obstetric and Gynecological Societies of India (FOGSI), National Neonatology Forum of India (NNF), and Indian Academy of Pediatrics (IAP). Indian Pediatr. 2020 Apr 01. Available from: www.indianpediatrics.net/CONVID29.03. 2020/RECOMM-00154.pdf. Accessed on April 06, 2020.

35. Landman A, Feetham L, Stuckey D. Cancer patients in SARS-CoV-2 infection: A nationwide analysis in China. Lancet Oncol. 2020;21:335-7.

36. Antiga LD. Coronaviruses and immunosuppressed patients. The facts during the third epidemic. AASLD. 2020 Mar 20. Available from: https:/laasldpubs. onlinelibrary.wiley.com/doi/epdf/10.1002/lt.25756. Accessed on April 05, 2020. [Epub ahead of print]

37. British Paediatric Allergy Immunity and Infection Group. BPAIIG Position Statement: Sars-CoV-2 Treatment Guidance version 1.2. Available from: https:// www.bpaiig.org/news-bpaiig-position-statement-sarscov-2-treatment-guidance-version-12. Accessed April 06, 2020 . 